\title{
Finite part of operator K-theory for groups with rapid decay
}

\author{
Sherry Gong
}

\begin{abstract}
In this paper we study the part of the $K$-theory of the reduced $C^{*}$-algebra arising from torsion elements of the group, and in particular we study the pairing of $K$-theory with traces and when traces can detect certain $K$-theory elements. In the case of groups with Property RD, we give a condition on the growth of conjugacy classes that determines whether they can be detected. Moreover, in the case that they can be detected, we show that nonzero elements in the part of the $K$-theory generated by torsion elements are not in the image of the assembly map $K_{0}^{G}(E G) \rightarrow K_{0}\left(C^{*} G\right)$. One application of this result is a lower bound for the structure groups of certain manifolds.
\end{abstract}

Mathematics Subject Classification (2010). 19-xx, 20-xx, 46-xx, 47-xx.

Keywords. Traces, unique tracial state, group $C^{*}$-algebras, reduced $C^{*}$-algebras, idempotents, operator $K$-theory, rapid decay property.

\section{Introduction}

Let $G$ be a group. An element $g$ is said to have order $d$ for positive integer $d$ if $g^{d}=1$ and $g^{k} \neq 1$ for any $0<k<d$. If no such $d$ exists, we say that $g$ has order $\infty$. We say an element is torsion if it has finite order.

For a torsion element $g$ of order $d$, we define a corresponding idempotent $p$ as

$$
p=\frac{1+g+g^{2}+\cdots+g^{d-1}}{d} \in \mathbb{C} G .
$$

It is easy to see that this is an idempotent, so we can view it in $K_{0}(\mathbb{C} G)$. Let $C_{\max }^{*} G$ denote the maximal group $C^{*}$-algebra and $C_{\text {red }}^{*} G$ denote the reduced group $C^{*}$ algebra. Then one can also view the above idempotent in $K_{0}\left(C_{\text {red }}^{*} G\right)$ or $K_{0}\left(C_{\max }^{*} G\right)$. The question we would like to study is whether for idempotents $p_{1}, \ldots p_{k}$ corresponding to torsion elements $g_{1}, \ldots, g_{k}$ of distinct orders $d_{1}, \ldots d_{k}>1$, we have that $1, p_{1}, \ldots p_{k}$ are linearly independent in $C_{\text {red }}^{*} G$ and $C_{\max }^{*} G$.

The study of idempotents arising from torsion elements was introduced in [8]. In that paper, Weinberger and Yu showed that if the group is finitely embeddable in Hilbert spaces, then the $p_{i}$ are linearly independent in $K_{0}\left(C_{\max }^{*}(G)\right)$. They 
conjectured that the same holds for all groups. They also showed that the rank of the part of $K_{0}\left(C_{\max }^{*}(G)\right)$ generated by these torsion elements bounds the non-rigidity for $M$. More precisely, it gives a lower bound for the rank of the structure group $S(M)$, that is, the abelian group of equivalence classes of pairs $\left(f, M^{\prime}\right)$, where $M^{\prime}$ is a compact, oriented manifold, and $f: M^{\prime} \rightarrow M$ is an orientation preserving homotopy equivalence.

Our approach to proving the linear independence of $p_{i}$ is to find $k+1$ traces on the algebra such that the $(k+1) \times(k+1)$ matrix that arises from evaluating the traces on $1, p_{1}, \ldots p_{k}$ has non-zero determinant. If there are such traces, then we say that $1, p_{1}, \ldots p_{k}$ can be distinguished by traces. It is easy to see that being distinguished by traces implies linear independence in $K_{0}$. For groups with rapid decay property (property RD), we prove a necessary and sufficient condition for the $1, p_{1}, \ldots p_{k} \in$ $K_{0}\left(C_{\text {red }}^{*} G\right)$ to be distinguished by traces, namely that they can be distinguished by traces if and only if the relevant conjugacy classes grow polyonimally. We discuss some examples of both our positive and our negative results. The negative results are particularly striking because they demonstrate a phenomenon in reduced group $C^{*}$-algebras that is very different from the maximal $C^{*}$-algebra case.

The author would like to thank Prof. Guoliang Yu for his helpful advice and guidance.

\section{Traces, idempotents, and rapid decay property}

In this section, we explain the conjecture of Weinberger and Yu in [8], introduce the method of using traces to detect idempotents, and define the rapid decay property.

Consider a group $G$ with torsion elements $g_{1}, g_{2}, \ldots, g_{k}$ of orders $d_{1}, \ldots d_{k}$ respectively, where the $d_{i}$ are distinct postive integers. For each $g_{i}$, we can consider the element

$$
p_{i}=\frac{1+g_{i}+g_{i}^{2}+\cdots+g_{i}^{d_{i}-1}}{d_{i}} \in \mathbb{C} G .
$$

Then $p_{i}$ is an idempotent, so we may consider it in the $K_{0}$ groups, $K_{0}\left(C_{\max }^{*}(G)\right)$ and $K_{0}\left(C_{\text {red }}^{*}(G)\right)$. We would like to explore whether

$$
p_{0}=1, p_{1}, \ldots p_{k}
$$

are linearly independent in these $K_{0}$ theories.

We call the subgroup of $K_{0}\left(C_{\max }^{*} G\right)$ (resp. $\left.K_{0}\left(C_{\text {red }}^{*} G\right)\right)$ generated by the $p_{i}$ the finite part of $K_{0}\left(C_{\max }^{*} G\right)$ (resp. $K_{0}\left(C_{\max }^{*} G\right)$ ).

It was conjectured by Weinberger and $\mathrm{Yu}$ in [8] that the $p_{i}$ are linearly independent in $K_{0}\left(C_{\max }^{*} G\right)$. More precisely:

Conjecture 2.1. For a group $G$ with torsion elements $g_{1}, g_{2}, \ldots, g_{k}$ of orders $d_{1}, \ldots d_{k}$ respectively, where the $d_{i}$ are distinct positive integers. Let $p_{i}$ denote 
the idempotent corresponding to $g_{i}$ as above. Then the $1, p_{1}, \ldots p_{k}$ are linearly independent in $K_{0}\left(C_{\max }^{*} G\right)$. Moreover, any nonzero element in the subgroup generated by $p_{1}, \ldots p_{k}$ is not in the image of the assembly map $K_{0}^{G}(E G) \rightarrow$ $K_{0}\left(C_{\max }^{*}(G)\right)$, where $E G$ is the universal space for proper and free $G$-action.

The assembly map is the map $K_{0}^{G}(E G) \rightarrow K_{0}\left(C_{\max }^{*}(G)\right)$ studied in [1] and [2].

To get at this question, we consider whether there are traces $\tau_{0}, \tau_{1}, \ldots, \tau_{k}$ : $C^{*} G: \rightarrow \mathbb{C}$ such that the matrix $A_{i j}=\tau_{i}\left(p_{j}\right)$ has non-zero determinant. If this were true, then we know that the $p_{i}$ are linearly independent, and we say that they are distinguishable by traces. If there are no such $\tau_{i}$, then the $p_{i}$ may still be linearly independent, but there is no way to prove this by traces, so we say that they are not distinguishable by traces.

Note that if idempotents can be separated by traces on $C_{\text {red }}^{*} G$, then they can also be separated by traces on $C_{\max }^{*} G$, because there is a natural map $C_{\max }^{*} G \rightarrow C_{\text {red }}^{*} G$, so the traces on $C_{\text {red }}^{*} G$ induce traces on $C_{\max }^{*} G$ in a way that does not affect the values on the idempotents. However, as we shall see, the converse is not true-indeed we shall give an example in which the idempotents can be separated by traces in $C_{\max }^{*}$, but not in $C_{\text {red }}^{*}$.

One other notion we will need is that of property RD. Recall that a length function on a group $G$ is a function $l: G \rightarrow \mathbb{Z}^{\geq 0}$, such that $l(f g) \leq l(f)+l(g)$, $l\left(g^{-1}\right)=l(g)$, and $l(e)=0$, such that for $S \subset \mathbb{Z}^{\geq 0}$ finite $l^{-1}(S)$ is also finite. Given such a length function on a group, there is a norm $\|\cdot\|_{H^{s}}$ on $\mathbb{C} G$ given by

$$
\left\|\sum c_{g} g\right\|_{H^{s}}^{2}=\sum\left|c_{g}\right|^{2}(1+l(g))^{2 s} .
$$

Let $H^{s}(G)$ denote the completion of $\mathbb{C} G$ with respect to this norm. Then we say $G$ has rapid decay property, or property $\mathrm{RD}$ if for some $s$, there is a length function on it and a constant $C$ such that

$$
\left\|\sum c_{g} g\right\|_{\text {red }} \leq C\left\|\sum c_{g} g\right\|_{H^{s}} .
$$

In this case, there is a natural map $H^{s} G \rightarrow C_{\text {red }}^{*} G$ obtained by extending the identity $\mathbb{C} G \rightarrow \mathbb{C} G$.

Note that for $t>s,\|x\|_{H^{t}}>\|x\|_{H^{s}}$ and we have natural map $H^{t}(G) \rightarrow$ $H^{s}(G)$. So if we have property RD for $s$, then we also have the inequality for any $t>s$.

Jolissaint introduced this property in [6]. He also showed in [7] that for such groups the map $H^{s} G \rightarrow C_{\text {red }}^{*} G$ induces and isomorphism of $K$-theory $K_{0}\left(H^{s} G\right) \rightarrow$ $K_{0}\left(C_{\text {red }}^{*} G\right)$.

\section{Results}

In this section, we show our main result, that in groups with property RD, the idempotents corresponding to torsion elements can be separated by traces if and 
only if the conjugacy classes of the torsion elements grow polynomially.

In Subsection 3.1 we show that idempotents can be separated by traces if the conjugacy classes grow polynomially, and in Section 3.2 we show the converse.

3.1. Positive Results. Let $G$ be a group with Property RD. For an element $g \in G$, let $C(g)$ denote its conjugacy class. Suppose that $g_{1}, \ldots g_{n} \in G$ are torsion elements such that $C\left(g_{i}\right)$ has polynomial growth, that is, for $C\left(g_{i}\right)_{l}=\left\{g \mid g \in C\left(g_{i}\right), l(g)=l\right\}$ and $n_{i, l}=\left|C\left(g_{i}\right)_{l}\right|$, we have $n_{i, l}<P(l)$ for some polynomial $P$. Then the $p_{i}$ can be separated by traces on $H^{s}(G)$ for some $s$. More precisely, we have:

Lemma 3.1. Let $G$ be a group with Property $R D$ and suppose $C\left(g_{1}\right)$ has polynomial growth. Then the trace $\tau: \mathbb{C} G \rightarrow G$ given by $\tau\left(\sum c_{g} g\right)=\sum_{g \in C\left(g_{1}\right)} c_{g}$ extends to $H^{s}(G)$ for sufficiently large $s$.

Proof. Notice that

$$
\begin{aligned}
\left|\sum_{g \in C\left(g_{1}\right)} c_{g}\right| \leq \sum_{l}\left|\sum_{g \in C\left(g_{1}\right)_{l}} c_{g}\right| & \leq \sum_{l} \sqrt{n_{1, l}} \sqrt{\sum_{g \in C\left(g_{1}\right), l}\left|c_{g}\right|^{2}} \\
& \leq C \sqrt{\sum_{l} n_{1, l}\left(\sum_{g \in C\left(g_{1}\right)_{l}}\left|c_{g}\right|^{2}\right) l^{2}}
\end{aligned}
$$

for some consant $C$. Here, the second inequality follows from the Power Mean Inequality, which says $\frac{\sum_{i=1}^{n} a_{i}}{n} \leq \sqrt{\frac{\sum a_{i}^{2}}{n}}$, and the third inequality follows from the Cauchy Schwarz inequality, which implies that for a sequence $a_{l}$,

$$
\sum a_{l} \leq \sqrt{\left(\sum a_{l}^{2} l^{2}\right)\left(\sum \frac{1}{l^{2}}\right)} \leq \frac{\pi}{\sqrt{6}} \sqrt{\left(\sum a_{l}^{2} l^{2}\right)} .
$$

But because $n_{1, l}$ is polynomial growth, the right hand side of (3.1) is bounded by the $H^{s}$ norm for some $s$, so the trace can be extended, as desired.

Theorem 3.2. Let $G$ be a group with Property $R D$. Let $g_{1}, \ldots g_{n}$ be torsion elements such that conjugacy classes $C\left(g_{i}\right)$ all have polynomial growth. Then for some s, the corresponding idempotents $p_{i}$ can be separated by traces on $H^{s}$. Thus, they are linearly independent in $K_{0}\left(H^{s} G\right)$. Moreover, they are linearly independent in $K_{0}\left(C_{r e d}^{*} G\right)$, and in $K_{0}\left(C_{r e d}^{*} G\right)$, nonzero elements in the subgroup generated by $p_{i}$ cannot lie in the image of the assembly map. Thus, groups with property $R D$ where all the conjugacy classes of torsion elements grow polynomially satisfy Conjecture 2.1. 
Proof. Consider $\tau_{0}: H^{s}(G) \rightarrow \mathbb{C}$ given by $\tau_{0}\left(\sum c_{g} g\right)=c_{e}$. This is clearly a bounded map. For $i=1, \ldots k$ consider $\tau_{i}: H^{s}(G) \rightarrow \mathbb{C}$ given as above by

$$
\tau_{i}\left(\sum c_{g} g\right)=\sum_{g \sim g_{i}} c_{g} .
$$

Then $\tau_{0}\left(p_{i}\right)=\frac{1}{d_{i}}$. Also note that for $d_{i}>d_{j}$, for any $t$, we have $\operatorname{deg}\left(g_{j}^{t}\right) \leq$ $\operatorname{deg}\left(g_{j}\right)<\operatorname{deg}\left(g_{i}\right)$, so $g_{j}^{t}$ and $g_{i}$ cannot be similar for any $j$. Thus for $d_{i}>d_{j}$, we have $\tau_{i}\left(p_{j}\right)=0$.

Also note that $\tau_{i}\left(p_{i}\right) \geq \frac{1}{d_{i}}$, because $\tau_{i}\left(g_{i}^{s}\right)$ is either 0 or 1 , and it is 1 for $s=1$.

So if we suppose that $d_{1}<d_{2}, \ldots$, we get that matrix $\tau_{i}\left(p_{j}\right)$ is upper triangular with all diagonal elements non-zero, so its determinant is nonzero, as desired.

Jolissaint showed in [7] that for property RD groups, the map $H^{s} G \rightarrow C_{\mathrm{red}}^{*} G$ is an isomorphism on the level of $K$-theory. That is, property RD implies $K_{0}\left(H^{s}(G)\right) \simeq K_{0}\left(C_{\text {red }}^{*}(G)\right.$. Thus, the $p_{i}$ are also linearly independent in $C_{\text {red }}^{*}(G)$.

Let us show that the non-zero elements in the subgroup generated by $p_{i}$ are not in the image of the assembly map $K_{0}^{G}(E G) \rightarrow K_{0}\left(C_{\text {red }}^{*} G\right)$. For a Hilbert space $H$, let $\mathcal{S}$ denote the ring of Schatten class operators, that is operators $T: H \rightarrow H$ such that there exists $p \geq 1$ such that $\operatorname{tr}\left(T^{*} T\right)^{p / 2}<\infty$, where the trace tr is the sum of the singular values. Let $\mathcal{S} G$ denote the group algebra of $G$ with coefficients in $\mathcal{S}$. As in [8] and [9], let $H_{0}^{\text {or } G}\left(E G, \mathbb{K}(\mathcal{S})^{-\infty}\right)$ be the generalized $G$-invariant homology theory associated to the non-connective algebraic K-theory spectrum $\left.\mathbb{K}(\mathcal{S})^{-\infty}\right)$. Then there is commutative diagram

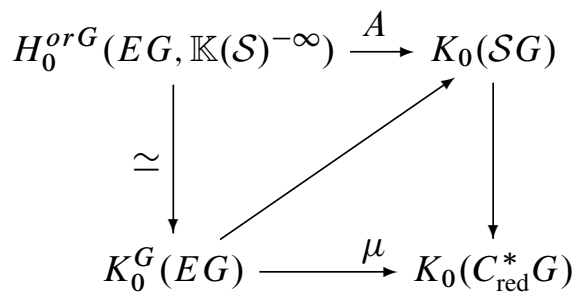

There is a homomorophism $j: \mathbb{C} G \rightarrow \mathcal{S} G$ given by $j(a)=P_{0} a$ where $P_{0}$ is a rank 1 projection. We may consider the finite part of $K_{0}(\mathcal{S} G)$ to be the subgroup generated by $j_{*}\left(p_{i}\right)$ where the $p_{i}$ are defined as above. To show that the finite part of $K_{0}\left(C_{\text {red }}^{*} G\right)$ is not in the image of $\mu$ it suffices to show that the finite part of $K_{0}(\mathcal{S} G)$ is not in the image of $H_{0}^{\text {or } G}\left(E G, \mathbb{K}(\mathcal{S})^{-\infty}\right)$.

For an algebra $\mathcal{A}$, we say that $\tau_{n}: \mathcal{A}^{\times n+1} \rightarrow \mathbb{C}$ is a multitrace if it satisfies

$$
\tau_{n}\left(a_{0}, a_{1}, \ldots a_{n}\right)=(-1)^{n} \tau_{n}\left(a_{1}, a_{2}, \ldots, a_{n}, a_{0}\right)
$$

and

$$
\sum_{i=0}^{n}(-1)^{i} \tau_{n}\left(a_{0}, \ldots, a_{i} a_{i+1}, \ldots a_{n+1}\right)+(-1)^{n+1} \tau_{n}\left(a_{n+1} a_{0}, a_{1}, \ldots a_{n}\right)=0 .
$$


For $g$ of finite order, consider the trace $\operatorname{tr}_{g}$ on $\mathcal{S}_{1} G$ given by $\operatorname{tr}_{g}\left(\sum s_{\gamma} \gamma\right)=$ $\sum_{\gamma \in C(g)} \operatorname{tr}\left(s_{\gamma}\right)$, where tr is the standard trace on $\mathcal{S}_{1}$. Then for $n=2 k$, consider $\tau_{n, g}$ on $S_{n}(G)$ given by

$$
\tau_{n, g}\left(a_{0}, \ldots a_{n}\right)=\operatorname{tr}_{g}\left(a_{0} a_{1} \cdots a_{n}\right) .
$$

It was shown in [8] (see the proof of Lemma 3.2 of [8]) that for element $p \in$ $K_{0}(\mathcal{S} G)$ in the image of $A$, we have that such $\tau_{n, g}, \tau_{n, g}(p, p, \ldots, p)=0$.

For groups with property $\mathrm{RD}$, we have commutative diagram

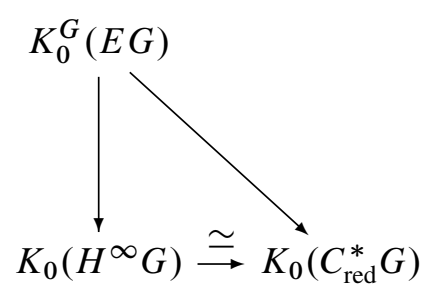

where $H^{\infty} G$ is the intersection of the $H^{s} G$, where the vertical maps are assembly maps.

Again as in [8], according to Connes' cyclic cohomology

$$
j^{*} \tau_{n, g}=S^{k} \tau_{g},
$$

where $\tau_{g}: \mathbb{C} G \rightarrow \mathbb{C}$ is defined by $\tau_{g}\left(\sum c_{\gamma} \gamma\right)=\sum_{\gamma \in C(g)} c_{\gamma}$. In [8] it was shown that for any element $x$ in the image of the assembly map $A$ : $H_{0}^{\text {or } G}\left(E G, \mathbb{K}(\mathcal{S})^{-\infty}\right) \rightarrow K_{0}(\mathcal{S} G)$, we have $\left\langle\tau_{n, g}, x\right\rangle=0$ for the $\tau_{n, g}$ as defined above, but in this case, we know that there is a trace $\tau_{g_{i}}$ (where $\left\{g_{i}\right\}$ is as in Conjecture 2.1) of this form that does not vanish on $x$, so $x$ cannot be in the image.

Now we show the conjecture, which is the same two statements, but for $C_{\max }^{*} G$ instead of $C_{\text {red }}^{*} G$. For the first, note that there is a natural map $C_{\max }^{*}(G) \rightarrow$ $C_{\text {red }}^{*}(G)$, so linear independence in $C_{\text {red }}^{*} G$ implies linear independence in $C_{\max }^{*}(G)$. For the second, note that the assembly map $K_{0}^{G}(E G) \rightarrow K_{0}\left(C_{\text {red }}^{*} G\right)$ factors through $K_{0}\left(C_{\max }^{*} G\right)$, so if the elements considered are in the image of $K_{0}^{G}(E G)$ in $K_{0}\left(C_{\max }^{*} G\right)$, then they are also in the image in $K_{0}\left(C_{\mathrm{red}}^{*} G\right)$.

Thus, we have shown Conjecture 2.1 for Property RD groups whose conjugacy classes of torsion elements have polynomial growth.

Example 3.3. Let us discuss some examples of groups which satisfy our conditions above. One example is groups of polynomial growth, which are known to have Property RD.

Another example is a polynomial-distortion extension of a torsion free word hyperbolic group by a polynomial growth group. That is we consider group $G$ with normal subgroup $N$ of polynomial growth such that $G / N$ is a torsion free word hyperbolic group and the length function of $G$ upon restriction to $N$ is polynomially distorted from the length function of $N$. 
It was shown in [6] that polynomial growth groups have property RD and in [3] that hyperbolic groups have property RD. Thus, by the following result of [5], $G$ has property RD.

Theorem 3.4. (Garncarek) Let $1 \rightarrow N \rightarrow G \rightarrow H \rightarrow 1$ be an extension with polynomial distortion. If $N$ and $H$ have property $R D$, then so does $G$.

All torsion elements in $G$ are contained in $N$, because $G / N$ is torsion-free. Thus, because the extension has polynomial distortion, the conjugacy classes of torsion elements have polynomial growth, and we may apply the above theorem.

Note that we have also established the conjecture for groups that are residually contained in the above class:

Definition 3.5. Let $\mathcal{R}$ be the class of groups $G$ with property RD such that for any torsion element $g, C(g)$ has polynomial growth. We say that a group $G$ is residually in $\mathcal{R}$ if for any finite subset $F \subset G$ there is a group $H \in \mathcal{R}$ and a homomorphism $\phi: G \rightarrow H$ such that $\phi$ is injective on $F$.

Theorem 3.6. Any group that is residually in $\mathcal{R}$ satisfies Conjecture 2.1.

Proof. Let $g_{1}, \ldots g_{k}$ be the torsion elements of order $d_{1}, \ldots d_{k}$ in question, and let $p_{1}, \ldots p_{k}$ be the corresponding idempotents. Let $F=\left\{g_{i}^{t_{i}} \mid 0 \leq t_{i}<d_{i}\right\}$. Then note that the $\phi\left(g_{i}\right)$ are torsion elements of order $d_{i}$ in $H$, and their corresponding idempotents in $\mathbb{C} H$ are $\phi\left(g_{i}\right)$, where $\phi$ denotes the induced map $\phi: \mathbb{C} G \rightarrow \mathbb{C} H$. But note that any unitary representation of $H$ is also a unitary representation of $G$, so $\phi$ also induces a map $C_{\max }^{*} G \rightarrow C_{\max }^{*} H$. By the above corollary, $H$ satisfies the conjecture, so for $p_{i} \in K_{0}\left(C_{\max }^{*} G\right)$, we have $\phi\left(p_{i}\right) \in K_{0}\left(C_{\max }^{*} H\right)$ are linearly independent. But this means that the $p_{i}$ are linearly independent in $K_{0}\left(C_{\max }^{*} G\right)$ as well.

The fact that nonzero elements in the subgroup generated by $p_{i}$ are not in the image of the assembly map follows from the functoriality of the assembly map.

This theorem has consequences related to structure groups of manifolds. In particular we have

Corollary 3.7. Let $M$ be a compact manifold of dimension $4 k-1$ with $k>1$ an integer and $\pi_{1}(M)=G$, and $G$ is in the class $\mathcal{R}$ described above. Then the rank of the structure group $S(M)$ is bounded below by the number of distinct orders of torsion elements of $G$.

Proof. This follows from Theorem 1.5 in [8], which states that this corollary holds for any $G$ satisfying Conjecture 2.1 
It is reasonable to conjecture that a similar statement to Conjecture 2.1 holds:

Conjecture 3.8. For a group $G$ with torsion elements $g_{1}, g_{2}, \ldots, g_{k}$ of orders $d_{1}, \ldots d_{k}$ respectively, where the $d_{i}$ are distinct positive integers. Let $p_{i}$ denote the idempotent corresponding to $g_{i}$. Then the $1, p_{1}, \ldots p_{k}$ are linearly independent in $K_{0}\left(C_{r e d}^{*} G\right)$. Moreover, any nonzero element in the subgroup generated by $p_{1}, \ldots p_{k}$ is not in the image of the assembly map $K_{0}^{G}(E G) \rightarrow K_{0}\left(C_{\max }^{*}(G)\right)$, where $E G$ is the universal space for proper and free $G$-action.

3.2. Negative Results. Now let us investigate the case where the group has Property RD, but some of the conjugacy classes do not have polynomial growth. We will show that all traces must be zero on these conjugacy classes.

Lemma 3.9. Let $G$ be a group of Property $R D$ and Let $h \in G$ be an element (which does not necessarily have to have finite order). Let $C(g)$ be its conjugacy class and let $C(g)_{l} \subset C(g)$ be those elements of length $l$, and let $n_{l}=\left|C(g)_{l}\right|$. Assume that $n_{l}$ is does not grow polynomially, that is, for any polynomial $P$, there are an infinite number of $l$ such that $n_{l}>P(l)$. Then any trace $\tau: C_{\text {red }}^{*} G \rightarrow \mathbb{C}$ must have $\tau(h)=0$.

Proof. The proof is in the same spirit as the proof in [6] that an amenable group that is not of polynomial growth does not have property RD. We proceed by contradiction. Suppose we have $\tau$ a trace such that $\tau(h) \neq 0$. By scaling it, we can assume $\tau(h)=1$. Then because $\tau$ is a trace, it is constant on conjugacy classes, so for any $h \in C(g)$, we have $\tau(h)=1$.

Since $n_{l}$ is super-polynomial, we may consider an increasing sequence $l_{1}, l_{2}, \ldots$ such that $n_{l_{i}}>\left(1+l_{i}\right)^{4 i}$. That is, $n_{l_{1}}>\left(1+l_{1}\right)^{4}, n_{l_{2}}>\left(1+l_{2}\right)^{8}$, etc.

Now consider the element $x=\sum x_{h} h$ with

$$
x_{h}=\left\{\begin{array}{ll}
n_{l(h)}^{-5 / 8} & l(h) \in\left\{l_{1}, l_{2}, \ldots\right\} \text { and } h \in C(g) \\
0 & \text { else }
\end{array} .\right.
$$

Then note that for the Sobolev norm $\left\|\sum c_{h} h\right\|_{H^{s}}^{2}=\sum\left|c_{h}\right|^{2}(1+l(h))^{2 s}$, we have that the above element is in $H^{s}$ with

$$
\left\|\sum x_{h} h\right\|_{H^{s}}=\sum_{i} n_{l_{i}} \cdot n_{l_{i}}^{-10 / 8}\left(1+l_{i}\right)^{2 s}<\sum_{i}\left(1+l_{i}\right)^{-i}\left(1+l_{i}\right)^{2 s}
$$

which clearly converges.

In fact, $x_{N}=\sum_{l(h)<N} x_{h} h$ converges to $x$ in the $H^{s}$ norm. Therefore, this is also true in the $C_{r}^{*} G$, because property RD.

However, I claim that for any $\tau$ with $\tau(h)=1$ on $C(g), \tau$ does not converge on $x_{N}$, therefore $\tau$ is not a bounded linear functional on $H^{s}$. Note that the value would be

$$
\sum_{i \text { with } l_{i}<N} n_{l_{i}} \cdot n_{l_{i}}^{-5 / 8}=\sum_{i \text { with } l_{i}<N} n_{l_{i}}^{3 / 8}
$$


but $n_{l_{i}}>1$ for all $i$, so clearly this sum diverges as $N \rightarrow \infty$.

Thus, $\tau$ cannot be a bounded linear functional.

Applying this lemma we have the following theorem:

Theorem 3.10. Let $G$ be a group with Property $R D$ and let $g_{1} \in G$ be a torsion element such that the conjugacy classes of $g_{1}^{t}$ do not grow polynomially for any $t$. Then in $C_{r e d}^{*}(G)$ we cannot distinguish $1, p_{1}$ by traces.

Proof. Applying the above lemma to each $g_{i}^{t}$ for $0<t<d$, we get that $\tau\left(g_{i}^{t}\right)=0$ for all $t$, which means that $\tau\left(p_{i}\right)=\tau\left(\frac{1}{d_{i}}\right)$, so for any trace $\tau$, we have $\tau\left(\left[p_{i}\right]\right)=$ $\frac{1}{d_{i}} \tau([1])$, so we cannot show that 1 and $p_{1}$ are linearly dependent using traces.

Note that a similar result does not hold for $C_{\max }^{*} G$. In particular, for torsion element $g \in G$ with order $d$, and $p$ the corresponding idempotent, we can always distinguish [1] and $[p]$ using the traces $\sum c_{g} g \mapsto c_{e}$ and $\sum c_{g} g \mapsto \sum c_{g}$, where the latter is a bounded map because it is the trace of the trivial action of $G$ on $\mathbb{C}$.

One example satisfying the above conditions is $G=<x, y>/ x^{3}$ with $g_{1}=x$. Then $G$ is a hyperbolic group, so it has Property RD. Also, $x$ and $x^{2}$ both have exponential growth conjugacy classes. In particular, the conjugacy classes contain $g x g^{-1}$ for

$$
g=y x^{a_{1}} y x^{3-a_{1}} y x^{a_{2}} y x^{3-a_{2}} y \cdots y x^{a_{l}} y x^{3-a_{l}} y,
$$

where $a_{i} \in\{1,2\}$. Then $g$ has length $5 l+1$ and $g x g^{-1}$ has length $10 l+3$, but there are $2^{l}$ choices of $g$. Thus, applying the above corollary, we see that [1] and $\left[p_{1}\right]$ are not distinguishable by traces.

We also have another corollary to the above lemma:

Theorem 3.11. Suppose that $G$ is a Property RD group such that none of its conjugacy classes grow polynomially. Then the only trace on $C_{r e d}^{*} G$ is $\sum c_{g} g \mapsto c_{e}$.

Remark 3.12. Note that this gives another proof of de la Harpe's results in [4] for unique trace on torsion-free non-elementary word hyperperbolic groups, that is, that such groups have a unique trace, because for such groups all conjugacy classes grow exponentially.

\section{References}

[1] A. Bartels, T. Farrell, L. Jones and H. Reich. On the isomorphism conjecture in algebraic $K$-theory. Topology 43 (2004), no. 1, 157-213. Zbl 1036.19003 MR 2030590

[2] P. Baum, A. Connes and N. Higson. Classifying space for proper actions and $K$-theory of group $C^{*}$-algebras in $C^{*}$-algebras: 1943-1993 (San Antonio, TX, 1993), 240-291, Contemp. Math., 167, Amer. Math. Soc., Providence, RI, 1994. Zbl 0830.46061 MR 1292018 
[3] P. de la Harpe, Groupes hyperboliques, algèbres d'opérateurs et un théorème de Jolissaint, C. R. Acad. Sci. Paris Sér. I Math. 307 (1988), no. 14, 771-774. Zbl 0653.46059 MR 972078

[4] P. de la Harpe. On operator algebras, free groups, and other groups. Astérisque 232 (S.M.F. 1995) 121-153. Zbl 0846.46039 MR 1372530

[5] Ł. Garncarek. A note on the property of rapid decay for group extensions, arXiv:1305.4872, 2014.

[6] P. Jolissaint. Rapidly decreasing functions in reduced $C^{*}$-algebras of groups. Trans. Amer. Math. Soc. 317 (1990), 167-196. Zbl 0711.46054 MR 943303

[7] P. Jolissaint. K-theory of reduced $C^{*}$-algebras and rapidly decreasing functions on groups. K-theory, (1989), no. 6, 723-735. Zbl 0692.46062 MR 1010979

[8] S. Weinberger and G. Yu. Finite part of operator K-theory for groups finitely embeddable into Hilbert space and the degree of non-rigidity of manifolds, arXiv:1308.4744, 2013.

[9] G. Yu. The Novikov conjecture for algebraic K-theory of the group algebra over the ring of Schatten class operators, arXiv:1106.3796, 2012.

Received 24 August, 2013; revised 06 January, 2014

S. Gong, Department of Mathematics, Massachusetts Institute of Technology,

77 Massachusetts Avenue, Cambridge, MA 02139, USA

E-mail:sgongli@math.mit.edu 